\title{
The impact of pathogen burden on leukocyte telomere length in the Multi-Ethnic Study of Atherosclerosis
}

\author{
A. E. AIELLO ${ }^{1 *}$, B. JAYABALASINGHAM ${ }^{2}$, A. M. SIMANEK ${ }^{3}$, A. DIEZ-ROUX ${ }^{4}$, \\ L. FEINSTEIN ${ }^{1,5}$, H. C. S. MEIER ${ }^{3}$, B. L. NEEDHAM ${ }^{6}$ AND J. B. DOWD ${ }^{2}$ \\ ${ }^{1}$ Department of Epidemiology, Gillings School of Global Public Health, and the Carolina Population Center, \\ The University of North Carolina at Chapel Hill, Chapel Hill, NC, USA \\ ${ }^{2}$ Department of Epidemiology and Biostatistics, CUNY School of Public Health, Hunter College, City University \\ of New York, USA \\ ${ }^{3}$ Joseph J. Zilber School of Public Health, University of Wisconsin-Milwaukee, Milwaukee, WI, USA \\ ${ }^{4}$ Dornsife School of Public Health, Drexel University, Philadelphia, PA, USA \\ ${ }^{5}$ Social \& Scientific Systems, Inc., Durham, NC, USA \\ ${ }^{6}$ Department of Epidemiology, Center for Social Epidemiology and Population Health, University of Michigan \\ School of Public Health, Ann Arbor, MI, USA
}

Received 28 March 2017; Final revision 2 August 2017; Accepted 2 August 2017; first published online 7 September 2017

\section{SUMMARY}

Several infections have been linked to telomere shortening and in some cases these associations have varied by sex. We assessed the association between seropositivity to four persistent pathogens (cytomegalovirus (CMV), herpes simplex virus-1, Helicobacter pylori, Chlamydia pneumoniae), and total pathogen burden on leukocyte telomere length in a diverse US sample. Data came from the Multi-Ethnic Study of Atherosclerosis, a population-based cohort study. We utilized cross-sectional survey data, and biological samples from participants tested for pathogens and telomere length $(N=163)$. Linear regression was used to examine the association between seropositivity for individual pathogens as well as total pathogen burden and telomere length, adjusting for various confounders. CMV seropositivity and increased total pathogen burden level were significantly associated with shorter telomere length among females $(\beta=-0 \cdot 1204$ (standard error (s.E.) $0 \cdot 06), P=0 \cdot 044)$ and $(\beta=-0 \cdot 1057$ (s.E. $=0 \cdot 05), P=0 \cdot 033$ ), respectively. There was no statistically significant association among males. Our findings suggest that prevention or treatment of persistent pathogens, in particular CMV, may play an important role in reducing telomere shortening over the life course among women. Future research is needed to confirm these novel findings in larger longitudinal samples.

Key words: Chlamydia pneumonia, cytomegalovirus, Helicobacter pylori, herpes simplex virus, pathogen burden, telomere.

\section{INTRODUCTION}

The mechanisms underlying inter-individual variation in telomere length are not well understood.

\footnotetext{
* Author for correspondence: A. E. Aiello, 135 Dauer Dr, 2101C McGavran-Greenberg Hall, Chapel Hill, NC 27599, USA. (Email: aaiello@unc.edu)
}

Telomeres are the nucleoprotein ends of chromosomes that function to protect chromosome ends from degradation or fusion and they shorten each time a cell divides, while the enzyme telomerase regulates the elongation process [1]. The process of telomere shortening has important health implications as reduced leukocyte telomere length has been linked to many chronic diseases of aging including 
cardiovascular disease and some cancers [2-5], as well as all-cause mortality [6].

Some preliminary studies have suggested that persistent herpesvirus infections, such as cytomegalovirus (CMV), and persistent bacterial infections including Helicobacter pylori (H. pylori) and Chlamydia pneumoniae (C. pneumoniae) may influence telomere length [7-9]. The mechanisms implicated are common across these pathogens, including induction of inflammation, generation of reactive oxygen species, and autoimmune changes - all of which may influence cellular machinery and ultimately telomere length [10].

Given that numerous persistent pathogens may contribute to physiologic processes related to telomere shortening, the total number of pathogens to which individuals have been exposed in their lifetime (i.e. total pathogen burden), may also play an important role in predicting telomere length. To our knowledge, no studies have examined the association between total pathogen burden and leukocyte telomere length.

It has been well established that female sex hormones influence telomere dynamics [11, 12]. At the same time, there is evidence that infectious disease susceptibility and immune response differ by sex, which may partly be driven by hormonal differences or gender disparate exposures, such as child care [13, 14]. In addition, previous work has shown an association between CMV infection and lower telomerase, an enzyme that helps maintain telomere length, in women but not in men [9]. Therefore, it is possible that sex (and/or gender) may modify the influence of pathogen burden on telomere length.

To address the gaps in research linking infections to telomere length in humans and potential variability in these associations by sex, we examined the association between seropositivity to four persistent pathogens (CMV, herpes simplex virus (HSV)-1, H. pylori and C. pneumoniae) as well as total pathogen burden and leukocyte telomere length among a sample of men and women participating in the Multi-Ethnic Study of Atherosclerosis (MESA).

\section{METHODS}

\section{Study population}

MESA is a longitudinal, US multi-site study of 6814 participants ages $45-84$ who were recruited from six US communities, were free of clinical cardiovascular disease at the time of the baseline visit from July 2000 to September 2002, and who identified themselves as white, black, Hispanic, or Chinese [15]. All individuals were tested for $C$. pneumoniae and a subset of 1000 participants were randomly selected from the baseline cohort for serotesting for CMV, HSV-1 and H. pylori, 999 of which had complete information on serostatus for all four pathogens [16]. Telomeres were assessed on a random subsample of approximately 1000 white, black, and Hispanic participants who agreed to participate in an ancillary study examining the effects of stress on cardiovascular outcomes (i.e. The MESA Stress Study) [17]. In total, 163 participants with overlapping data on pathogens and telomere length were included in the present study, which was reviewed by the Institutional Review Board of the University of Michigan and at each MESA site.

\section{Measures}

Exposures

Serum IgG antibodies to CMV, HSV-1, and H. pylori were detected using commercially available kits, employing an indirect enzyme immunoassay (DiaMedex Corp., Miami, Florida, USA). The sensitivity and specificity of the tests ranged from $94 \%$ to 100\% (DiaMedex Corp). IgG antibodies to C. pneumoniae were detected using a microimmunoflourescent antibody assay (Focus Technologies, Cypress, California, USA). Serum IgG antibodies for each pathogen were treated as continuous or dichotomized according to the below cutoff values. Individuals were classified as CMV seronegative, equivocal, or seropositive if values were $<8 \cdot 0$, between $8 \cdot 0$ and $9 \cdot 0$, or $10 \cdot 0+$ ELISA Units $(\mathrm{EU}) / \mathrm{ml}$, respectively. Cutoff values for being classified as HSV-1 seronegative, equivocal, and seropositive were $<16 \cdot 0,16 \cdot 0-19 \cdot 9$, and $20 \cdot 0+\mathrm{EU} / \mathrm{ml}$, respectively. For H. pylori, EU $/ \mathrm{ml}$ values of $<0.90$ were classified as negative, 0.90-1.09 as equivocal, and $\geqslant 1 \cdot 10$ as positive. All individuals with equivocal values $(\mathrm{CMV} ; N=0, \mathrm{HSV}-1 ; N=0$, H. pylori; $N=6, C$. pneumoniae; $N=0$ ) were categorized as seropositive. Total pathogen burden level was constructed by summing the number of pathogens for which individuals were seropositive and then dichotomizing individuals into low/reference group (seropositive for $0-2$ pathogens) and high (seropositive for 3-4 pathogens) total pathogen burden level. These cut points were based on prior literature and the need to ensure adequate sample size among the reference group (low category) [18, 19]. 


\section{Outcome}

Leukocyte telomere length was measured by quantitative polymerase chain reaction (Q-PCR) performed using DNA isolated from purified leukocytes [20]. A four-point standard curve (twofold serial dilutions from 10 to $1.25 \mathrm{ng}$ DNA) was used to transform cycle threshold into nanograms of DNA. Baseline background subtraction was performed by aligning amplification plots to a baseline height of $2 \%$ in the first five cycles. The cycle threshold was set at $20 \%$ of maximum product. All samples were run in triplicate, and the median was used for calculations. The amount of telomeric DNA $(T)$ was divided by the amount of single-copy control gene (36B4) DNA $(S)$, producing a normalized measurement of leukocyte telomere length ( $T / S$ ratio). Two control samples were run in each experiment to allow for normalization between experiments, and periodical reproducibility experiments were performed to guarantee correct measurements. The intra-assay and inter-assay coefficient of variability for Q-PCR was 6\% and 7\%, respectively. Leukocyte telomere length was treated as continuous.

\section{Covariates}

Socio-demographic, behavioral, and clinical information was collected via questionnaire at baseline. Demographic covariates for which data were collected and were hypothesized to be potential confounders of interest included age, sex, race/ethnicity, and socioeconomic status. Race/ethnicity was self-reported as non-Hispanic black, Hispanic, and non-Hispanic white. Annual family income was categorized as <US $\$ 25000, \$ 25000-\$ 50000$, and $>\$ 50000$ and education level was categorized as high school or less; some college (including Associate's Degree or technical school); or bachelor's or graduate degree. In addition, hypothesized behavioral and clinical confounders were pack-years of smoking (the number of packs of cigarettes smoked per day times the number of years the person reporting smoking), body mass index (BMI) $\left(\mathrm{kg} / \mathrm{m}^{2}\right)$ calculated from measured weight and height, and diabetes history. Diabetes history was assessed according to the 2003 American Diabetes Association criteria and categorized as normal, impaired fasting glucose (IFG), untreated diabetes, or treated diabetes [21, 22].

\section{Statistical analysis}

We first examined the association between seropositivity for individual pathogens as well as total pathogen burden and covariates of interest. For continuous, normally distributed characteristics (i.e. leukocyte telomere length and $\mathrm{BMI}), t$ tests were used to detect statistically significant differences for mean values between groups. For continuous, but not normally distributed characteristics (i.e. age and pack-years of smoking) Wilcoxon rank-sum tests were performed and medians and interquartile ranges (IQRs) were estimated for each group. Fisher's exact tests were used to test for significant differences in proportions for categorical characteristics to accommodate small cell sizes.

We used linear regression to first estimate the association between seropositivity for each individual pathogen and telomere length adjusting for age, sex, race/ethnicity, and education level. Next, we additionally adjusted for pack-years of smoking, BMI $\left(\mathrm{kg} / \mathrm{m}^{2}\right)$, and diabetes history. Analogous models were run for the association between total pathogen burden and telomere length. We repeated all models stratified by sex and also ran models including an interaction term between sex and the primary exposure (i.e. pathogen seropositivity or total pathogen burden). Analyses were performed using PROC MIXED in SAS to adjust for clustering by MESA sites (SAS Institute Inc., Cary, North Carolina, USA).

\section{RESULTS}

Table 1 shows the demographic and health characteristics of our study population by pathogen burden level. A higher proportion of blacks, participants with lower education, pack-years of smoking, and hormone use were associated with higher pathogen burden. The unadjusted associations between participant characteristics and telomere length are shown in Table 2. In the full sample, individuals seropositive to CMV, HSV-1, and C. pneumonia as well as those with high pathogen burden had shorter mean telomere lengths than those who were seronegative to these pathogens or had low total pathogen burden, respectively, but no statistically significant associations were observed. Of the other covariates of interest, only increased age was statistically significantly associated with shorter telomere length $(\beta=-0.0029$ (standard error, s.E. $=0 \cdot 0014), P$-value 0.0400). Among females, increased pack-years of smoking was statistically significantly associated with shorter telomere length $(\beta=-0.0041 \quad$ (s.e. $=0.0018), \quad P$-value 0.025$), \quad$ and there was a marginally statistically significant association between increasing BMI $\left(\mathrm{kg} / \mathrm{m}^{2}\right)(\beta=-0 \cdot 0045$ 
Table 1. Selected characteristics of the Multi-Ethnic Study of Atherosclerosis (MESA) study sample by pathogen burden level

\begin{tabular}{|c|c|c|c|}
\hline & \multicolumn{2}{|c|}{ Mean (S.E. $)^{\mathrm{a}}$, median $(\mathrm{IQR})^{\mathrm{b}}$, or $N(\%)^{\mathrm{c}}$} & \multirow[b]{2}{*}{$P$-value } \\
\hline & $\begin{array}{l}\text { Low pathogen burden } \\
(N=34)\end{array}$ & $\begin{array}{l}\text { High pathogen burden } \\
(N=129)\end{array}$ & \\
\hline Telomere length $^{\mathrm{a}}$ & $0 \cdot 917(0 \cdot 159)$ & $0 \cdot 900(0 \cdot 166)$ & $0 \cdot 6$ \\
\hline Age in years ${ }^{\mathrm{b}}$ & $58(50-66)$ & $55(51-63)$ & 0.472 \\
\hline \multicolumn{4}{|l|}{$\operatorname{Sex}^{\mathrm{c}}$} \\
\hline Female & $17(50)$ & $83(64 \cdot 3)$ & \multirow[t]{2}{*}{$0 \cdot 127$} \\
\hline Male & $17(50)$ & $46(35 \cdot 7)$ & \\
\hline \multicolumn{4}{|l|}{ Race/ethnicity ${ }^{\mathrm{c}}$} \\
\hline White & $17(50)$ & $18(14)$ & \multirow[t]{3}{*}{$<0 \cdot 001$} \\
\hline Black & $3(8 \cdot 8)$ & $38(29 \cdot 5)$ & \\
\hline Hispanic & $14(41 \cdot 2)$ & $73(56 \cdot 6)$ & \\
\hline \multicolumn{4}{|l|}{ Education $^{\mathrm{c}}$} \\
\hline Complete HS/GED or less & $5(14 \cdot 7)$ & $68(52 \cdot 7)$ & \multirow[t]{3}{*}{$<0 \cdot 001$} \\
\hline Some college & $14(41 \cdot 2)$ & $39(30 \cdot 2)$ & \\
\hline Bachelor's Degree or more & $15(44 \cdot 1)$ & $22(17 \cdot 1)$ & \\
\hline \multicolumn{4}{|l|}{ Income $^{\mathrm{c}}$} \\
\hline$<\$ 5000-\$ 24999$ & $7(20 \cdot 6)$ & $40(32 \cdot 8)$ & \multirow[t]{3}{*}{$0 \cdot 063$} \\
\hline$\$ 25000-\$ 49999$ & $10(29 \cdot 4)$ & $47(38 \cdot 5)$ & \\
\hline$\$ 50000-\$ 100000+$ & $17(50)$ & $35(28 \cdot 7)$ & \\
\hline Pack-years of smoking ${ }^{\mathrm{b}}$ & $4 \cdot 3(0-15 \cdot 0)$ & $0(0-3 \cdot 8)$ & 0.005 \\
\hline BMI $\left(\mathrm{kg} / \mathrm{m}^{2}\right)^{\mathrm{a}}$ & $28 \cdot 1(5 \cdot 8)$ & $29 \cdot 1(5 \cdot 5)$ & $0 \cdot 351$ \\
\hline \multicolumn{4}{|l|}{ Diabetes mellitus $^{\mathrm{c}}$} \\
\hline Normal & $28(82 \cdot 4)$ & $104(80 \cdot 6)$ & \multirow[t]{3}{*}{0.717} \\
\hline IFG & $2(5 \cdot 9)$ & $13(10 \cdot 1)$ & \\
\hline Untreated/treated DM & $4(11 \cdot 8)$ & $12(9 \cdot 3)$ & \\
\hline \multicolumn{4}{|l|}{ Hormone medication use $^{\mathrm{c}}$} \\
\hline No & $23(67 \cdot 7)$ & $106(82 \cdot 8)$ & \multirow[t]{2}{*}{$0 \cdot 051$} \\
\hline Yes & $11(32 \cdot 4)$ & $22(17 \cdot 2)$ & \\
\hline
\end{tabular}

${ }^{\mathrm{a}} T$ test (mean and s.E. displayed).

${ }^{\mathrm{b}}$ Wilcoxon rank-sum test (median and IQR displayed).

${ }^{\mathrm{c}}$ Fisher's exact test ( $n$ and $\%$ displayed).

$($ s.e. $=0.0025), P$-value $=0.068), \mathrm{CMV}$ seropositivity $(\beta=-0.0998 \quad$ (s.E. $=0.0548), \quad P$-value 0.0720$)$ and shorter telomere length. Among males, the only covariate that was statistically significantly associated with shorter telomere length was education level, with individuals with some college education having statistically significantly shorter telomere length than those that completed high school/GED or less $(\beta=-$ $0 \cdot 1282$ (s.E. $=0 \cdot 0496), P$-value $=0 \cdot 012$ ).

Table 3 shows the covariate-adjusted associations between pathogen seropositivity as well as total pathogen burden level and telomere length among the total sample and stratified by sex. Among the full sample, there were no statistically significant associations between seropositivity for individual pathogens or total pathogen burden level and telomere length. In stratified models, however, there was a statistically significant association between CMV seropositivity and telomere length among women after adjusting for age, race/ethnicity, education level, pack-years of smoking, BMI $\left(\mathrm{kg} / \mathrm{m}^{2}\right)$, and diabetes history $(\beta=-$ $0 \cdot 1204$ (s.E. $=0 \cdot 06$ ),$\quad P$-value $=0 \cdot 044$ ). Higher total pathogen burden level was also statistically significantly associated with shorter telomere length among women only $(\beta=-0 \cdot 1057 \quad$ (s.E. $=0 \cdot 05), \quad P$-value $=$ 0.033 ) in the fully adjusted model. There was a marginally statistically significant interaction between CMV seropositivity and sex $(P=0.08)$ and between total pathogen burden level and sex $(P=0 \cdot 11)$.

\section{DISCUSSION}

To our knowledge, this is the first study to examine the association between seropositivity for a wide 
Table 2. Mean difference in leukocyte telomere length for selected characteristics of the Multi-Ethnic Study of Atherosclerosis (MESA) study sample, overall and stratified by gender

\begin{tabular}{|c|c|c|c|c|c|c|}
\hline & \multicolumn{2}{|c|}{ Total sample $(n=163)$} & \multicolumn{2}{|l|}{ Female $(n=100)$} & \multicolumn{2}{|l|}{ Male $(n=63)$} \\
\hline & Mean diff. (s.E.) & $P$-value & Mean diff. (s.E.) & $P$-value & Mean diff. (s.E.) & $P$-value \\
\hline Age in years & $-0.0029(0 \cdot 0014)$ & $0 \cdot 04$ & $-0 \cdot 0030(0 \cdot 0018)$ & $0 \cdot 094$ & $-0 \cdot 0028(0 \cdot 0023)$ & $0 \cdot 23$ \\
\hline \multicolumn{7}{|l|}{ Sex } \\
\hline Male & REF & & & & & \\
\hline Female & $0 \cdot 0118(0 \cdot 0265)$ & $0 \cdot 656$ & & & & \\
\hline \multicolumn{7}{|l|}{ Race/ethnicity } \\
\hline White & REF & & REF & & REF & \\
\hline Black & $-0 \cdot 0167(0 \cdot 0381)$ & $0 \cdot 662$ & $-0 \cdot 0350(0 \cdot 0482)$ & $0 \cdot 47$ & $0 \cdot 0270(0 \cdot 0622)$ & $0 \cdot 666$ \\
\hline Hispanic & $-0 \cdot 0123(0 \cdot 0331)$ & $0 \cdot 712$ & $0 \cdot 0234(0 \cdot 0430)$ & $0 \cdot 587$ & $-0.0673(0.0517)$ & $0 \cdot 198$ \\
\hline \multicolumn{7}{|l|}{ Education } \\
\hline Complete HS/GED or less & REF & & REF & & REF & \\
\hline Some college & $-0 \cdot 0450(0 \cdot 0297)$ & $0 \cdot 131$ & $0 \cdot 0056(0 \cdot 0368)$ & $0 \cdot 878$ & $-0 \cdot 1282(0 \cdot 0496)$ & $0 \cdot 012$ \\
\hline Bachelor's Degree or more & $-0.0213(0 \cdot 0332)$ & $0 \cdot 521$ & $0 \cdot 0108(0 \cdot 0425)$ & $0 \cdot 8$ & $-0.0754(0 \cdot 0533)$ & $0 \cdot 162$ \\
\hline \multicolumn{7}{|l|}{ Income } \\
\hline$<\$ 5000-\$ 24999$ & $0 \cdot 0233(0 \cdot 0334)$ & $0 \cdot 486$ & $-0 \cdot 0087(0 \cdot 0426)$ & $0 \cdot 838$ & $0 \cdot 0611(0 \cdot 0597)$ & $0 \cdot 311$ \\
\hline$\$ 25000-\$ 49999$ & $0.0030(0.0319)$ & 0.926 & $-0 \cdot 0290(0 \cdot 0417)$ & $0 \cdot 488$ & $0 \cdot 0370(0 \cdot 0529)$ & $0 \cdot 488$ \\
\hline$\$ 50000-\$ 100000+$ & REF & & REF & & REF & \\
\hline Pack-years of smoking & $-0 \cdot 0009(0 \cdot 0011)$ & $0 \cdot 387$ & $-0 \cdot 0041(0 \cdot 0018)$ & $0 \cdot 025$ & $0 \cdot 0007(0 \cdot 0014)$ & $0 \cdot 605$ \\
\hline BMI $\left(\mathrm{kg} / \mathrm{m}^{2}\right)$ & $-0 \cdot 0030(0 \cdot 0023)$ & $0 \cdot 196$ & $-0 \cdot 0045(0 \cdot 0025)$ & $0 \cdot 068$ & $0 \cdot 0029(0 \cdot 0059)$ & $0 \cdot 619$ \\
\hline \multicolumn{7}{|l|}{ Diabetes mellitus } \\
\hline Normal & REF & & REF & & REF & \\
\hline IFG & $-0 \cdot 0125(0 \cdot 0450)$ & $0 \cdot 781$ & $-0 \cdot 0138(0 \cdot 0593)$ & $0 \cdot 816$ & $-0 \cdot 0066(0 \cdot 0701)$ & 0.926 \\
\hline Untreated/treated DM & $0.0396(0.0437)$ & $0 \cdot 366$ & $-0.0066(0.0537)$ & $0 \cdot 902$ & $0 \cdot 1164(0 \cdot 0751)$ & $0 \cdot 127$ \\
\hline \multicolumn{7}{|l|}{ Hormone medication use } \\
\hline No & REF & & REF & & REF & \\
\hline Yes & $0 \cdot 0195(0 \cdot 0322)$ & $0 \cdot 547$ & $0 \cdot 0249(0 \cdot 0342)$ & $0 \cdot 469$ & $-0 \cdot 1853(0 \cdot 1757)$ & $0 \cdot 296$ \\
\hline \multicolumn{7}{|l|}{ Pathogen burden } \\
\hline Low $(0-2)$ & REF & & REF & & REF & \\
\hline High (3-4) & $-0 \cdot 0167(0 \cdot 0318)$ & 0.6 & $-0 \cdot 0567(0 \cdot 0421)$ & $0 \cdot 181$ & $0 \cdot 0245(0 \cdot 0498)$ & $0 \cdot 624$ \\
\hline \multicolumn{7}{|l|}{ CMV serostatus } \\
\hline Negative & REF & & REF & & REF & \\
\hline Positive & $-0 \cdot 0267(0 \cdot 0352)$ & $0 \cdot 449$ & $-0 \cdot 0998(0 \cdot 0548)$ & $0 \cdot 072$ & $0 \cdot 0121(0 \cdot 0499)$ & $0 \cdot 809$ \\
\hline \multicolumn{7}{|l|}{ HSV serostatus } \\
\hline Negative & REF & & REF & & REF & \\
\hline Positive & $-0 \cdot 0095(0 \cdot 0461)$ & $0 \cdot 836$ & $-0 \cdot 1058(0 \cdot 0807)$ & $0 \cdot 193$ & $0 \cdot 0273(0 \cdot 0605)$ & 0.654 \\
\hline \multicolumn{7}{|l|}{ Helicobacter pylori serostatus } \\
\hline Negative & $\mathrm{REF}$ & & REF & & $\mathrm{REF}$ & \\
\hline Positive & $0 \cdot 0087(0 \cdot 0269)$ & $0 \cdot 747$ & $0 \cdot 0139(0 \cdot 0334)$ & 0.679 & $-0 \cdot 0002(0 \cdot 0456)$ & 0.997 \\
\hline \multicolumn{7}{|c|}{ Chlamydia pneumoniae serostatus } \\
\hline Negative & REF & & REF & & REF & \\
\hline Positive & $-0.0393(0 \cdot 0285)$ & $0 \cdot 17$ & $-0.0635(0 \cdot 0330)$ & $0 \cdot 058$ & $0 \cdot 0199(0 \cdot 0564)$ & $0 \cdot 725$ \\
\hline
\end{tabular}

S.E., standard error.

array of persistent pathogens as well as total pathogen burden and telomere length. We identified a stronger association between CMV seropositivity as well as higher total pathogen burden level and telomere length among females compared with males, suggesting a sex-specific association of persistent pathogens on telomere shortening. Taken together, seropositivity for persistent pathogens may be a particularly salient risk factor for cellular aging among women.

Few studies have examined the association between individual persistent pathogens and leukocyte telomere length [4, 23-25]. Van de Berg et al. showed that CMV seropositivity was associated with T-cell telomere shortening in a cohort of 159 healthy volunteers $20-95$ years of age and attributed this finding to 
Table 3. Covariate-adjusted association between pathogen serostatus, pathogen burden, and leukocyte telomere length, overall and stratified by gender

\begin{tabular}{|c|c|c|c|c|c|c|c|c|c|c|c|c|c|c|c|}
\hline & \multicolumn{5}{|c|}{ Total sample $(N=163)$} & \multicolumn{5}{|c|}{ Women $(N=100)^{\mathrm{c}}$} & \multicolumn{5}{|c|}{$\operatorname{Men}(N=63)^{\mathrm{c}}$} \\
\hline & \multirow[b]{2}{*}{$N$} & \multicolumn{2}{|l|}{ Model $1^{\mathrm{a}}$} & \multicolumn{2}{|l|}{ Model $2^{b}$} & \multicolumn{3}{|c|}{ Model $1^{\mathrm{a}}$} & \multicolumn{2}{|l|}{ Model $2^{\mathrm{b}}$} & \multicolumn{3}{|c|}{ Model $1^{\mathrm{a}}$} & \multicolumn{2}{|l|}{ Model $2^{\mathrm{b}}$} \\
\hline & & $\beta$ (S.E.) & $P$-value & $\beta$ (S.E.) & $P$-value & $N$ & $\beta$ (S.E.) & $P$-value & $\beta$ (S.E.) & $P$-value & $N$ & $\beta$ (S.E.) & $P$-value & $\beta$ (S.E.) & $P$-value \\
\hline \multicolumn{16}{|l|}{ CMV } \\
\hline Negative & 26 & REF & & REF & & 9 & REF & & REF & & 17 & REF & & REF & \\
\hline Positive & 137 & $\begin{array}{c}-0.0400 \\
(0 \cdot 04)\end{array}$ & $0 \cdot 291$ & $\begin{array}{c}-0 \cdot 0377 \\
(0 \cdot 04)\end{array}$ & 0.323 & 91 & $\begin{array}{c}-0 \cdot 1209 \\
(0 \cdot 06)\end{array}$ & 0.042 & $\begin{array}{c}-0 \cdot 1204 \\
(0 \cdot 06)\end{array}$ & $0 \cdot 044$ & 46 & $\begin{array}{c}-0.0039 \\
(0 \cdot 05)\end{array}$ & $0 \cdot 940$ & $\begin{array}{c}-0 \cdot 0112 \\
(0 \cdot 05)\end{array}$ & 0.827 \\
\hline \multicolumn{16}{|l|}{ HSV-1 } \\
\hline Negative & 14 & REF & & REF & & 4 & REF & & REF & & 10 & REF & & REF & \\
\hline Positive & 149 & $\begin{array}{c}-0.0038 \\
(0.05)\end{array}$ & 0.938 & $0 \cdot 0008(0 \cdot 05)$ & 0.987 & 96 & $\begin{array}{c}-0 \cdot 1034 \\
(0 \cdot 0820)\end{array}$ & $0 \cdot 211$ & $\begin{array}{c}-0 \cdot 1057 \\
(0 \cdot 08)\end{array}$ & $0 \cdot 211$ & 53 & $\begin{array}{r}0.0389 \\
(0 \cdot 06)\end{array}$ & 0.537 & $\begin{array}{r}0 \cdot 0498 \\
(0 \cdot 06)\end{array}$ & $0 \cdot 416$ \\
\hline \multicolumn{16}{|l|}{$\begin{array}{l}\text { Helicobacter } \\
\text { pylori }\end{array}$} \\
\hline Negative & 59 & REF & & REF & & 35 & REF & & REF & & 24 & REF & & REF & \\
\hline Positive & 104 & $\begin{array}{c}-0.0039 \\
(0.03)\end{array}$ & 0.890 & $\begin{array}{c}-0 \cdot 0108 \\
(0 \cdot 03)\end{array}$ & $0 \cdot 706$ & 65 & $\begin{array}{r}0 \cdot 0042 \\
(0 \cdot 04)\end{array}$ & $0 \cdot 909$ & $\begin{array}{c}-0 \cdot 0156 \\
(0 \cdot 04)\end{array}$ & 0.683 & 39 & $\begin{array}{r}0.0064 \\
(0 \cdot 04)\end{array}$ & $0 \cdot 883$ & $\begin{array}{c}-0 \cdot 0132 \\
(0 \cdot 04)\end{array}$ & 0.761 \\
\hline \multicolumn{16}{|l|}{$\begin{array}{l}\text { Chlamydia } \\
\text { pneumonia }\end{array}$} \\
\hline Negative & 46 & REF & & REF & & 34 & REF & & REF & & 12 & REF & & REF & \\
\hline Positive & 117 & $\begin{array}{c}-0.0470 \\
(0.03)\end{array}$ & $0 \cdot 121$ & $\begin{array}{c}-0.0412 \\
(0 \cdot 03)\end{array}$ & $0 \cdot 182$ & 66 & $\begin{array}{c}-0.0469 \\
(0.04)\end{array}$ & $0 \cdot 190$ & $\begin{array}{c}-0 \cdot 0495 \\
(0 \cdot 04)\end{array}$ & $0 \cdot 178$ & 51 & $\begin{array}{c}-0 \cdot 0271 \\
(0 \cdot 05)\end{array}$ & 0.617 & $\begin{array}{r}0 \cdot 0011 \\
(0 \cdot 05)\end{array}$ & $0 \cdot 984$ \\
\hline \multicolumn{16}{|c|}{ Pathogen burden } \\
\hline $0-2$ & 34 & REF & & REF & & 17 & REF & & REF & & 17 & REF & & REF & \\
\hline $3-4$ & 129 & $\begin{array}{c}-0.0354 \\
(0.04)\end{array}$ & $0 \cdot 314$ & $\begin{array}{c}-0 \cdot 0367 \\
(0 \cdot 0354)\end{array}$ & $0 \cdot 301$ & 83 & $\begin{array}{c}-0 \cdot 0648 \\
(0 \cdot 05)\end{array}$ & $0 \cdot 163$ & $\begin{array}{c}-0 \cdot 1057 \\
(0 \cdot 05)\end{array}$ & $0 \cdot 033$ & 46 & $\begin{array}{r}0 \cdot 0095 \\
(0 \cdot 05)\end{array}$ & $0 \cdot 858$ & $\begin{array}{r}0 \cdot 0091 \\
(0 \cdot 05)\end{array}$ & 0.859 \\
\hline
\end{tabular}

${ }^{a}$ Adjusted for age, race/ethnicity, sex, education level.

${ }^{\mathrm{b}}$ Adjusted for age, race/ethnicity, sex, education level, pack-years of smoking, BMI $\left(\mathrm{kg} / \mathrm{m}^{2}\right)$, and diabetes history.

${ }^{\mathrm{c}}$ Sex was not included in the sex-stratified model. 
CMV seropositive individuals having increased proportions of highly differentiated CD4+ and CD8+ T cells [26]. A similar study by Dowd et al. found no association between CMV seropositivity or IgG antibody level and telomere length among 434 adult men and women in the Whitehall II cohort [9]. However, similar to our findings, Dowd et al. observed a statistically significant inverse association between CMV seropositivity as well as elevated CMV IgG antibody level and telomerase activity among females compared with males [9]. We are aware of only one study conducted among a clinical population that has examined the association between H. pylori and leukocyte telomere length [4]. In a casecontrol study of gastric cancer conducted among 300 cases and 416 age- and sex-matched controls in Poland, Hou et al. found among controls that $H$. pylori seropositivity was statistically significantly associated with shorter telomere length in peripheral leukocyte DNA [4]. In another study Aslan et al. assessed change in gastric mucosal tissue telomere length and telomerase activity before and after $\mathrm{H}$. pylori eradication treatment among $21 \mathrm{H}$. pylori-infected individuals, finding a statistically significant increase in telomere length in gastric mucosa after treatment [27]. While $H$. pylori seropositivity was associated with shorter leukocyte telomere length in our study, the association did not reach statistical significance possibly due to limited statistical power in our sample.

To our knowledge, no other studies have examined the relationship between HSV-1, C. pneumoniae seropositivity nor pathogen burden and leukocyte telomere length. While we did not observe an association between these less well-studied individual pathogens and telomere shortening in the total sample, we did find that increased total pathogen burden level was statistically significantly associated with shorter telomere length among women. Given the association between pathogen burden and telomere length identified here, our findings suggest that there may be a cumulative impact of increased total pathogen burden on telomere shortening, particularly among women. Further research assessing the mechanisms by which pathogen burden may influence telomere shortening and differences in these associations by sex is warranted.

Interestingly, while the severity and prevalence of most viruses is higher among males, Herpesviridae family viruses, including CMV, Human Herpes Virus Type 6 (HHV-6), Human Herpes Virus Type 7 (HHV-7), Varicella Zoster Virus (VZV) and
HSV-2 appear to be exceptions [28, 29]. Some have suggested that sex hormones and chromosomes underlie these differences. For example, the female sex hormone estrogen serves to enhance T-cell-mediated immune processes, potentially via the presence of the estrogen response element in the promoters of many of the upregulated inflammation genes [30]. In vitro studies suggest that estradiol may trigger the reactivation of CMV and cervical shedding of CMV has been shown to increase during the luteal phase of the menstrual cycle and in later stages of pregnancy - both of which are dominated by progesterone production [3133]. Together, these hormone-driven differences in immunity and maintenance of latency may be responsible for the observations in population-based studies that women are not only more likely to be seropositive for CMV but to also have elevated levels of circulating CMV IgG antibodies, compared with men [34, 35]. Given the well-established effects of female sex hormones on telomere dynamics, further research examining this shared pathway is warranted [11, 12]. Furthermore, future studies should aim to elucidate the biological mechanisms by which exposure to multiple persistent pathogens over the life course may be particularly detrimental for telomere shortening among women.

There are several reasons why CMV, more so than other pathogens, may contribute to telomere shortening. CMV contributes to oligoclonal T-cell expansion resulting in the accumulation of highly differentiated late-stage CD8+ $\mathrm{T}$ cells specific for CMV [36]. For this reason, $\mathrm{CMV}$ in particular, may be an important driver of replicative senescence within the T-cell compartment, particularly among those who undergo more frequent subclinical reactivation over time [37]. The pathogen-specific interaction of CMV with the stress hormone cortisol - shown to inhibit telomerase activity in CD4 and CD8 T cells [38] - may also explain why CMV, more so than other pathogens, contributes to telomere shortening. For example, CMV can infect and replicate in human adrenocortical cells, thereby triggering steroidogenesis [39]. Moreover, there are many CMV strains and individuals can be re-infected throughout the course of their lifetime [40-42]. However, much of the incidence of infection occurs early in life and therefore it is unlikely that new CMV infection fully explains our results [43].

This study has several limitations. Given the lack of overlap of those tested for both telomere length and pathogen seropositivity among participants in MESA, our sample size was limited $(n=163)$, and 
may have reduced our ability to detect statistically significant associations and sex-specific interactions. Given that coefficients were consistently in the expected direction in the overall sample with pathogen seropositivity and higher total pathogen burden predicting shorter leukocyte telomere length, further analysis with larger samples are warranted. The cross-sectional nature of our study did not permit assessment of the timing of initial infection nor the effect of pathogen seropositivity and total pathogen burden on changes in telomere length over time. Additionally, measurement of the shortest telomere and telomere uncapping have been suggested as more accurate measures of senescence, but these methods have not been widely adopted in epidemiological studies because of the expense related to conducting these assays on a large scale [3, 44]. Nonetheless, to our knowledge, our study represents the largest study to date to examine the association between multiple persistent pathogens and leukocyte telomere length.

Overall, our study confirms earlier findings, which suggest that CMV seropositivity may play an important role in telomere shortening, particularly among women and moreover, some evidence that there may be a cumulative effect of exposure to CMV in the presence of other persistent pathogens on telomere length. Our results suggest that if persistent pathogens are causally related to telomere shortening, treatments to manage reactivation or prevent infection through the development of vaccinations may serve to decrease telomere attrition across the life course. Future research corroborating these findings in larger population-based studies is therefore warranted.

\section{ACKNOWLEDGEMENTS}

The authors thank the other investigators, the staff, and the participants of the MESA study for their valuable contributions. A full list of participating MESA investigators and institutions can be found at http:// www.mesa-nhlbi.org.

Allison Aiello and Jennifer Beam Dowd were supported by NIH Grant R01AG040115. Allison Aiello and Lydia Feinstein were supported by the Population Research Infrastructure Program (P2C HD050924) awarded to the Carolina Population Center at The University of North Carolina at Chapel Hill by the Eunice Kennedy Shriver National Institute of Child Health and Human Development. Lydia Feinstein was supported by the Population Research Training grant (T32
HD007168) awarded to the Carolina Population Center at The University of North Carolina at Chapel Hill by the Eunice Kennedy Shriver National Institute of Child Health and Human Development. Ana Diez-Roux was supported by NIH Grant R01 HL101161.

This research was supported by contracts HHSN268201500003I, N01-HC-95159, N01-HC-95160, N01-HC-95161, N01-HC-95162, N01-HC-95163, N01HC-95164, N01-HC-95165, N01-HC-95166, N01-HC95167, N01-HC-95168, and N01-HC-95169 from the National Heart, Lung, and Blood Institute; and by grants UL1-TR-000040, UL1-TR-001079, and UL1-TR001420 from NCATS.

\section{DISCLAIMERS}

None.

\section{DECLARATION OF INTEREST}

None.

\section{REFERENCES}

1. Olovnikov AM. A theory of marginotomy. The incomplete copying of template margin in enzymic synthesis of polynucleotides and biological significance of the phenomenon. Journal of Theoretical Biology 1973; 41 (1): 181-190.

2. Kroenke CH, et al. Telomerase, telomere length, and coronary artery calcium in black and white men in the CARDIA study. Atherosclerosis 2012; 220(2): 506-512.

3. Sanders JL, et al. Leukocyte telomere length is associated with noninvasively measured age-related disease: The Cardiovascular Health Study. The Journals of Gerontology Series A, Biological Sciences and Medical Sciences 2012; 67(4): 409-416.

4. Hou L, et al. Telomere length in peripheral leukocyte DNA and gastric cancer risk. Cancer Epidemiology, Biomarkers \& Prevention: A Publication of the American Association for Cancer Research, Cosponsored by the American Society of Preventive Oncology 2009; 18(11): 3103-3109.

5. Wentzensen IM, et al. The association of telomere length and cancer: a meta-analysis. Cancer Epidemiology, Biomarkers \& Prevention: A Publication of the American Association for Cancer Research, Cosponsored by the American Society of Preventive Oncology 2011; 20(6): 1238-1250.

6. Deelen $\mathbf{J}$, et al. Leukocyte telomere length associates with prospective mortality independent of immune-related parameters and known genetic markers. International Journal of Epidemiology 2014; 43(3): 878-886.

7. O'Donovan A, et al. Cumulative inflammatory load is associated with short leukocyte telomere length in the 
Health, Aging and Body Composition Study. Public Library of Science One 2011; 6(5): e19687.

8. Wong JY, et al. The relationship between inflammatory biomarkers and telomere length in an occupational prospective cohort study. Public Library of Science One 2014; 9(1): e87348.

9. Dowd JB, et al. Cytomegalovirus is associated with reduced telomerase activity in the Whitehall II cohort. Experimental Gerontology 2013; 48(4): 385-390.

10. Effros RB. Telomere/telomerase dynamics within the human immune system: effect of chronic infection and stress. Experimental Gerontology 2011; 46(2-3): 135-140.

11. Gladych MM, Wojtyla AA, Rubis BB. Human telomerase expression regulation. Biochemistry and Cell Biology 2011; 89(4): 359-376.

12. Barrett ELB, Richardson DS. Sex differences in telomeres and lifespan. Aging Cell 2011; 10(6): 913-921.

13. Giefing-Kroll C, et al. How sex and age affect immune responses, susceptibility to infections, and response to vaccination. Aging Cell 2015; 14(3): 309-321.

14. Klein SL, Marriott I, Fish EN. Sex-based differences in immune function and responses to vaccination. Transactions of the Royal Society of Tropical Medicine and Hygiene 2015; 109(1): 9-15.

15. Bild DE. Multi-Ethnic Study of Atherosclerosis: objectives and design. American journal of epidemiology 2002; 156(9): 871-881.

16. Aiello AE, et al. Socioeconomic and psychosocial gradients in cardiovascular pathogen burden and immune response: the Multi-Ethnic Study of Atherosclerosis. Brain, Behavior, and Immunity 2009; 23(5): 663-671.

17. Diez Roux AV, et al. Race/ethnicity and telomere length in the Multi-Ethnic Study of Atherosclerosis. Aging Cell 2009; 8(3): 251-257.

18. Zhu J, et al. Effects of total pathogen burden on coronary artery disease risk and C-reactive protein levels. American Journal of Cardiology 2000; 85(2): 140-146.

19. Lutsey PL, et al. Serological evidence of infections and type 2 diabetes: the Multi-Ethnic Study of Atherosclerosis. Diabetic Medicine 2009; 26(2): 149-152.

20. Cawthon RM. Telomere measurement by quantitative PCR. Nucleic acids research 2002; 30(10): e47.

21. American Diabetes Association. Diagnosis and Classification of Diabetes Mellitus. 2004, pp. S5-S10. Available at: http:// care.diabetesjournals.org/content/27/suppl_1/s5.long

22. Golden SH, et al. Depression and type 2 diabetes mellitus: the Multi-Ethnic Study of Atherosclerosis. Psychosomatic Medicine 2007; 69(6): 529-536.

23. Hearps AC, et al. HIV infection and aging of the innate immune system. Sexual Health 2011; 8(4): 453-464.

24. Kitay-Cohen Y, et al. Telomere length in Hepatitis C. Cancer Genetics and Cytogenetics 2008; 187(1): 34-38.

25. Zhang Y, et al. Telomere length in peripheral blood lymphocytes contributes to the development of HPV-associated oropharyngeal carcinoma. Cancer Research 2013; 73(19): 5996-6003.

26. van de Berg PJ, et al. Cytomegalovirus infection reduces telomere length of the circulating $\mathrm{T}$ cell pool. Journal of Immunology 2010; 184(7): 3417-3423.
27. Aslan R, et al. Helicobacter pylori eradication increases telomere length in gastric mucosa. HepatoGastroenterology 2013; 60(123): 601-604.

28. Klein SL. Sex influences immune responses to viruses, and efficacy of prophylaxis and treatments for viral diseases. BioEssays 2012; 34(12): 1050-1059.

29. Muenchhoff M, Goulder PJR. Sex differences in pediatric infectious diseases. The Journal of Infectious Diseases 2014; 209(Suppl. 3): S120-S126.

30. Hewagama A, et al. Stronger inflammatory/cytotoxic $\mathrm{T}$-cell response in women identified by microarray analysis. Genes and Immunity 2009; 10(5): 509-516.

31. Mostad SB, et al. Cervical shedding of herpes simplex virus and cytomegalovirus throughout the menstrual cycle in women infected with human immunodeficiency virus type 1. American Journal of Obstetrics and Gynecology 2000; 183(4): 948-955.

32. Shen CY, et al. Cytomegalovirus excretion in pregnant and nonpregnant women. Journal of Clinical Microbiology 1993; 31(6): 1635-1636.

33. Stagno S, et al. Cervical cytomegalovirus excretion in pregnant and nonpregnant women: suppression in early gestation. Journal of Infectious Disease 1975; 131 (5): 522-527.

34. Staras SA, et al. Seroprevalence of cytomegalovirus infection in the United States, 1988-1994. Clinical Infectious Diseases 2006; 43(9): 1143-1151.

35. Dowd JB, Aiello AE. Socioeconomic differentials in immune response. Epidemiology 2009; 20(6): 902-908.

36. Pawelec G, et al. Cytomegalovirus and human immunosenescence. Reviews in Medical Virology 2009; 19(1): 47-56.

37. Dock JN, Effros RB. Role of CD8T cell replicative senescence in human aging and in HIV-mediated immunosenescence. Aging and Disease 2011; 2(5): 382-397.

38. Choi J, Fauce SR, Effros RB. Reduced telomerase activity in human T lymphocytes exposed to cortisol. Brain, Behavior, and Immunity 2008; 22(4): 600-605.

39. Trevisan M, et al. Human cytomegalovirus productively infects adrenocortical cells and induces an early cortisol response. Journal of Cellular Physiology 2009; 221(3): 629-641.

40. Bale JF Jr., et al. Cytomegalovirus reinfection in young children. The Journal of Pediatrics 1996; 128(3): 347-352.

41. Gaytant MA, et al. Congenital cytomegalovirus infection after recurrent infection: case reports and review of the literature. European Journal of Pediatrics 2003; 162(4): 248-253.

42. Novak Z, et al. Cytomegalovirus strain diversity in seropositive women. Journal of Clinical Microbiology 2008; 46(3): 882-886.

43. Colugnati FA, et al. Incidence of cytomegalovirus infection among the general population and pregnant women in the United States. BioMed Central Infectious Diseases 2007; 7: 71.

44. Chebel A, et al. Telomere uncapping during in vitro T-lymphocyte senescence. Aging Cell 2009; 8(1): 52-64. 